\title{
ПРИБЛИЖЕННЫЕ АЛГОРИТМЫ С ГАРАНТИРОВАННЫМИ ОЦЕНКАМИ ТОЧНОСТИ ДЛЯ ПЕРЕСЕЧЕНИЯ МНОЖЕСТВ РЕБЕР НЕКОТОРЫХ МЕТРИЧЕСКИХ ГРАФОВ РАВНЫМИ КРУГАМИ ${ }^{1}$
}

\author{
К. С. Кобылкин
}

\begin{abstract}
В статье предлагаются полиномиальные приближенные алгоритмы с константным фактором аппроксимации для задачи пересечения заданного набора $n$ отрезков на плоскости наименьшим числом одинаковых кругов. Если число различных направлений отрезков, входящих в набор, не превосходит $k$, для этой задачи известен простой $4 k$-приближенный алгоритм с временной сложностью $O(n \log n)$. Также для случая задачи на множествах ребер произвольных плоских графов известен $100-$ приближенный алгоритм с временем работы $O\left(n^{4} \log n\right)$. В настоящей работе для частных случаев задачи на множествах ребер графов Габриеля, графов относительных окрестностей и минимальных евклидовых остовных деревьев, число различных ориентаций ребер в которых, вообще говоря, не ограничено сверху, удается построить несложные алгоритмы с факторами аппроксимации, равными соответственно 14, 12 и 10, имеющие существенно меньшую трудоемкость $O\left(n^{2}\right)$ по сравнению с вышеупомянутым алгоритмом для общего случая задачи на множествах ребер произвольных плоских графов.
\end{abstract}

Ключевые слова: комбинаторная оптимизация, приближенный алгоритм, геометрическая задача Нitting Set на плоскости, прямолинейный отрезок, граф Габриеля, граф относительных окрестностей, минимальное евклидово остовное дерево.

K. S. Kobylkin. Approximation algorithms with guaranteed performance for the intersection of edge sets of some metric graphs with equal disks.

Polynomial-time approximation algorithms with constant approximation ratio are proposed for the problem of intersection of a given set of $n$ planar straight line segments with the least number of equal disks. In the case where the segments have at most $k$ different orientations, a simple $4 k$-approximate algorithm with time complexity $O(n \log n)$ is known. In addition, a 100-approximate algorithm with time $\operatorname{complexity~} O\left(n^{4} \log n\right)$ is known for the case of the problem on the edge sets of plane graphs. In this paper, for instances of the problem on the edge sets of Gabriel graphs, relative neighbourhood graphs, and Euclidean minimum spanning trees, in which the number of different edge orientations is, in general, unbounded, we construct simple $O\left(n^{2}\right)$-time approximation algorithms with approximation ratios 14, 12, and 10, respectively. These algorithms outperform the aforementioned approximation algorithm for the general setting of the problem for edge sets of plane graphs.

Keywords: combinatorial optimization, approximation algorithm, geometric Hitting Set problem on the plane, straight line segment, Gabriel graph, relative neighborhood graph, Euclidean minimum spanning tree.

MSC: $90 \mathrm{C} 15$

DOI: $10.21538 / 0134-4889-2019-25-1-62-77$

\section{Введение}

Задачи оптимального размещения геометрических объектов простой формы на плоскости являются важным классом задач, лежащим на стыке вычислительной геометрии и комбинаторной оптимизации. В классической постановке задач этого класса требуется разместить фиксированное число объектов, скажем производства, склады или центры продажи товаров таким образом, чтобы среднее (или максимальное) расстояние от клиента (например, от потребителя или от участка дороги) до ближайшего к нему объекта было бы минимально. При этом множеству возможных размещений объектов соответствует множество $F$, состоящее из фигур на плоскости простой формы, скажем, кругов или прямоугольников, а множеству клиентов отвечает конечное множество $K$ из точек или отрезков. В более сложных постановках

\footnotetext{
${ }^{1}$ Исследования поддержаны Российским научным фондом, грант № 14-11-00109.
} 
требуется оптимизировать сумму транспортных затрат и стоимости размещения объектов. В других, также ставших классическими постановках задач размещения, требуется покрыть или пересечь элементы $K$ минимальным числом объектов из $F$. Постановки такого типа называются в литературе задачами оптимального покрытия. Такие постановки возникают в задачах оптимального размещения супермаркетов в непосредственной близости от потенциальных покупателей, а также в задачах мониторинга дорожных сетей с помощью одинаковых сенсоров с ограниченной областью охвата (sensing scope).

В работе даются полиномиальные приближенные алгоритмы с константным фактором аппроксимации для следующей NP-трудной задачи, являющейся представителем подкласса задач размещения, формулируемых в виде задач оптимального геометрического покрытия:

З а д а ч а Intersecting Plane Graph with Disks (IPGD): для заданных $r>0$ и укладки $G=(V, E)$ простого (т. е. без петель и кратных ребер) планарного графа с $n$ прямолинейными ребрами, которые могут пересекаться не более чем в своих концевых точках, найти наименьшее по мощности подмножество $H \subset \mathbb{R}^{2}$ точек (центров кругов) с условием, что всякое ребро $e \in E$ находится на евклидовом расстоянии, не превосходящем $r$, от некоторой точки $x=x(e) \in H$; другими словами, круг радиуса $r$ с центром в точке $x$ пересекает ребро $e$. Всякая изолированная вершина $v \in V$ при этом трактуется как отрезок $e_{v} \in E$ нулевой длины, а множество $V$ предполагается находящимся в общем положении.

В нашем изложении при обозначении сформулированной задачи оптимального пересечения отрезков будет использоваться аббревиатура IPGD, а для обозначения укладки графа термин "плоский граф".

Одним из важных приложений данной постановки является мониторинг дорожной сети с помощью одинаковых сенсорных устройств, имеющих круговую область охвата. Обеспечение полного покрытия всех участков дорожной сети областями охвата сенсоров может быть очень дорогим и энергозатратным мероприятием. При этом с точки зрения безопасности может оказаться достаточно охватить лишь некоторую часть каждого участка дороги с помощью одного из размещенных сенсоров. Если в качестве оптимизируемого критерия взять число используемых сенсоров и разбить дорожную сеть на прямолинейные участки без перекрестков, такую задачу можно сформулировать с виде некоторой постановки задачи IPGD.

В работе полиномиальный по вычислительной сложности и затратам памяти алгоритм (обозначим его через $\mathcal{A}$ ) для задачи IPGD называется $f$-приближсенным (или имеющим фактор аппроксимации $f$ ), если для всякого плоского графа $G$, принадлежащего некоторому классу графов, равномерно внутри этого класса выполнено следующее неравенство:

$$
\frac{\left|H_{\mathcal{A}}(G, r)\right|}{O P T(G, r)} \leq f
$$

где $H_{\mathcal{A}}(G, r)$ - допустимое решение задачи IPGD, выдаваемое алгоритмом $\mathcal{A}$ для заданного графа $G$ и радиуса $r, O P T=O P T(G, r)$ - оптимум задачи IPGD для данного графа $G$ и радиуса $r$.

Пусть $\alpha$ и $\beta$ - вычислимые функции натурального аргумента $m$. Всюду ниже также используется стандартное обозначение $\alpha(m)=O(\beta(m))$, равносильное существованию такой константы $D>0$, что для всех достаточно больших $m$ справедливо неравенство $|\alpha(m)| \leq D|\beta(m)|$.

\section{1. Приближенные алгоритмы для задачи IPGD: известные и новые алгоритмические результаты}

Задача IPGD обобщает классическую задачу оптимального покрытия заданного конечного множества точек $E$ на плоскости одинаковыми кругами на случай, когда отрезки в $E$ имеют ненулевую длину, и, как следствие, является NP-трудной ([6;9]). По-видимому, впервые, задача, близкая к IPGD, рассматривалась в работе [6]. В этой работе для множества $E$, вообще говоря, попарно пересекающихся по относительной внутренности отрезков, каждый из которых 
имеет направление, параллельное произвольной из двух осей фиксированной прямоугольной системы координат, построен 8-приближенный алгоритм с временными затратами $O(n \log n)$ и затратами памяти $O(n \log n)$. Данный алгоритм можно легко обобщить до $4 k$-приближенного на случай не более чем $k$ ориентаций. Для общего случая сколь угодно большого числа различных ориентаций отрезков из $E$ при допущении их попарного пересечения в точках относительной внутренности представляется маловероятным [2] существование полиномиального приближенного алгоритма с константным фактором аппроксимации, по крайней мере, на основе известных алгоритмических парадигм. Однако, если отрезкам позволяется пересекаться не более чем в своих концевых точках ${ }^{2}$, известен 100-приближенный алгоритм [10] со сложностью $O\left(n^{4} \log n\right)$ и затратами памяти $O\left(n^{2} \log n\right)$. При этом существование $O(1)$-приближенного алгоритма для этой задачи с существенно большим 100 константным фактором аппроксимации следует из результатов более ранней работы [14].

Задача IPGD является частным случаем хорошо исследованной классической задачи НітTING SET о наименьшей системе представителей на плоскости. Некоторые результаты об аппроксимируемости последней переносятся также и на задачу IPGD. В общем случае задача HitTing SET формулируется следующим образом: требуется найти такое подмножество $H$ заданного множества $Y \subseteq \mathbb{R}^{2}$, что $H \cap R \neq \varnothing$ для любого $R \in \mathcal{R}$, где $\mathcal{R}$ - заданное семейство подмножеств в $\mathbb{R}^{2}$, называемых также объектами. В дальнейшем подмножество $H \subset \mathbb{R}^{2}$ называется системой представителей для $\mathcal{R}$, если $H \cap R \neq \varnothing$ для любого $R \in \mathcal{R}$. Кроме того, со всякой постановкой задачи НIтTING SEт связывается упорядоченная пара множеств $(Y, \mathcal{R})$, называемая пространством.

Очевидно, задача IPGD эквивалентна задаче HitTing SET, заданной на пространстве $\left(\mathbb{R}^{2}, \mathcal{N}_{r}(E)\right)$, где $\mathcal{N}_{r}(E)=\left\{N_{r}(e): e \in E\right\}, N_{r}(e)=\left\{x \in \mathbb{R}^{2}: d(x, e) \leq r\right\}$ и $d(x, e)$ - евклидово расстояние между точкой $x \in \mathbb{R}^{2}$ и отрезком $e \in E$. Также можно доказать, что $\mathcal{N}_{r}(E)$ можно без ограничения общности считать семейством выпуклых псевдодисков, под которым понимается такое конечное семейство выпуклых замкнутых компактов с непустой внутренностью на плоскости, что две замкнутых жордановых кривых на границе всякой пары множеств этого семейства пересекаются не более чем дважды и дополнение любого множества в этой паре до другого является связным. Отсюда следует, что для задачи IPGD существует PTAS [13] (см. также [12] и введение в [5]) очень высокой сложности. Отсюда также вытекает существование 4-приближенного алгоритма [3] для задачи IPGD с временной сложностью $O\left(n^{18}\right)$.

Таким образом, для общей постановки задачи IPGD в классе произвольных плоских графов у известных на данный момент приближенных алгоритмов относительно высокая точность, т. е. близкий к 1 константный фактор аппроксимации, достигается лишь при очень большой трудоемкости; и, наоборот, за приемлемое время может быть достигнута лишь невысокая точность. В связи с этим обстоятельством возникает необходимость в построении достаточно точных приближенных алгоритмов невысокой трудоемкости для задачи IPGD в классах специальных плоских графов, возникающих в разного рода приложениях. В данной статье удается достигнуть хорошего сочетания характеристик точности и трудоемкости у предлагаемых приближенных алгоритмов для задачи IPGD на некоторых специальных классах плоских графов, называемых в литературе метрическими (proximity graphs). Предлагаемые алгоритмы являются концептуально гораздо более простыми по сравнению с вышеупомянутым 100приближенным алгоритмом для общей постановки задачи на множествах ребер произвольных плоских графов.

Дадим несколько определений. Пусть $V$ - множество точек в общем положении на плоскости. В предположении, что никакие четыре точки из $V$ не лежат на одной окружности, будем называть плоский граф $G=(V, E)$ графом Габриеля [11] при условии, что $[u, v] \in E$ тогда и только тогда, когда внутренность круга с диаметром $[u, v]$ не содержит точек множества $V$. При том же предположении будем называть плоский граф $G=(V, E)$ графом относителъных

\footnotetext{
${ }^{2}$ что совпадает с общей постановкой задачи IPGD в классе произвольных плоских графов
} 
окрестностей [8], если $[u, v] \in E$ тогда и только тогда, когда $\max \{d(u, w), d(v, w)\} \geq d(u, v)$ для любого $w \in V \backslash\{u, v\}$.

Плоский граф $G=(V, E)$ называется минимальным евклидовым остовным деревом, если этот граф совпадает с остовным деревом минимального веса для полного взвешенного графа, вершины которого находятся в точках множества $V$, а веса ребер задаются евклидовыми расстояниями между их концевыми вершинами. Можно показать, что всякий граф Габриеля на множестве $V$ содержит в себе некоторый граф относительных окрестностей на множестве $V$, который, в свою очередь, содержит в себе некоторое минимальное евклидово остовное дерево на $V$.

Все вышеперечисленные типы графов возникают во множестве приложений в анализе физических сетей. Например, такие графы представляют из себя удобные сетевые топологии, упрощающие маршрутизацию и контроль в географических (в частности, в беспроводных) сетях.

В [9] для $r>0$ показана NP-трудность задачи IPGD, рассматриваемой на любом из трех перечисленных классов графов. В данной работе предлагается 14-приближенный алгоритм для этой задачи в классе графов Габриеля, 12-приближенный алгоритм - в классе графов относительных окрестностей и 10-приближенный алгоритм - для класса минимальных евклидовых остовных деревьев. Эти алгоритмы имеют одинаковые по порядку временные затраты $O(n O P T)$ и пространственные затраты $O(n)$. При этом изложенный в работе алгоритм для графов Габриеля имеет лучшее сочетание характеристик точности и сложности по сравнению с таковым для алгоритма, описанного в [10].

\section{2. Основные идеи предлагаемых приближенных алгоритмов}

Идеи, лежащие в основе предлагаемых в данной работе $O(1)$-приближенных алгоритмов, можно суммировать в следующих двух определениях и опирающемся на них алгоритме.

О п р е д е л е н и е 1 . Подмножество $\mathcal{I} \subseteq \mathcal{N}_{r}(E)$ называется максимальным (по включению) независимым множеством в $\mathcal{N}_{r}(E)$, если $I \cap I^{\prime}=\varnothing$ для всяких $I, I^{\prime} \in \mathcal{I}$, и для каждого $N \in \mathcal{N}_{r}(E)$ найдется такой $I \in \mathcal{I}$, что $N \cap I \neq \varnothing$.

О п р е д е л е н и е 2. Пусть $G=(V, E)$ - плоский граф, а $C>0$ - заданная абсолютная константа. Будем говорить, что ребро $e \in E$ является $C$-покрываемым относительно $E$, если при любом $r>0$ для семейства $\mathcal{N}_{e}(E)=\left\{N \in \mathcal{N}_{r}(E): N \cap N_{r}(e) \neq \varnothing\right\}$ можно за полиномиальное время (по $\left.\left|\mathcal{N}_{e}(E)\right|\right)$ построить систему представителей $U(e, E) \subset \mathbb{R}^{2}$ мощности, не превосходящей $C$.

Рассмотрим следующий основной алгоритм.

А Л Г О Р И Т М ПОКРЫТИЕ РЕБЕР СПЕЦИАЛЬНЫХ ГРАФОВ РАВНЫМИ КРУГАМИ

Вход: $r>0$ и плоский граф $G=(V, E)$.

Выход: приближенное решение $H$ задачи IPGD для графа $G$ и числа $r$.

1. $E^{\prime}:=\varnothing, E_{0}:=E$ и $H:=\varnothing$.

2. До тех пор, пока $E_{0} \neq \varnothing$, повторять шаги 3-4.

3. Выбрать произвольное $C$-покрываемое ребро $e^{*} \in E_{0}$ (относительно $E_{0}$ ) и построить систему представителей $U\left(e^{*}, E_{0}\right)$ мощности, не большей $C$, для семейства $\mathcal{N}_{e^{*}}\left(E_{0}\right)$ с помощью некоторой вспомогательной процедуры.

4. Положить $E_{0}:=E_{0} \backslash\left\{e \in E_{0}: N_{r}(e) \cap U\left(e^{*}, E_{0}\right) \neq \varnothing\right\}, E^{\prime}:=E^{\prime} \cup\left\{e^{*}\right\}, H:=H \cup U\left(e^{*}, E_{0}\right)$.

5. Вернуть множество $H$.

Для того, чтобы полностью описать работу данного выше алгоритма для некоторого класса плоских графов $\mathcal{G}$, необходимо для всякого подмножества $E_{0} \subseteq E$ во множестве ребер $E$ произвольного графа из $\mathcal{G}$ указать (конструктивный) способ выбора $C$-покрываемого ребра $e^{*} \in E_{0}$. Кроме того, для произвольного выбираемого этим способом ребра $e \in E_{0}$ следует описать вспомогательную процедуру поиска системы представителей $U\left(e, E_{0}\right)$ семейства $\mathcal{N}_{e}\left(E_{0}\right)$, 
имеющей длину, не большую $C$. Предполагается, что такая процедура должна принимать на вход ребро $е$ и семейство $\mathcal{N}_{e}\left(E_{0}\right)$.

В дальнейшем обозначение $\mathcal{N}_{e}\left(E_{0}\right)$ будет использоваться только в ситуациях, когда нужно подчеркнуть, что в семейство $\mathcal{N}_{e}\left(E_{0}\right)$ входят имеющие непустое пересечение с объектом $N_{r}(e)$ $r$-окрестности тех отрезков, которые принадлежат некоторому, в частности собственному, подмножеству $E_{0} \subseteq E$. Если же $E_{0}=E$, будет использоваться более простое обозначение $\mathcal{N}_{e}$.

В приводимом ниже утверждении описывается достаточное условие, при котором алгоритм ПОКРЫТИЕ РЕБЕР СПЕЦИАЛЬНЫХ ГРАФОВ РАВНЫМИ КРУГАМИ ЯВЛЯеТСЯ $C$-ПриблИЖеННЫМ.

Утверждение 1. Пусть $C>0$ - некоторая константа и класс $\mathcal{G}$ плоских графов таков, что для любого $G=(V, E) \in \mathcal{G}$ и всякого $E_{0} \subseteq E$ за полиномиальное время $O\left(\varphi\left(\left|E_{0}\right|\right)\right)$ с линейными пространственными затратами найдется ребро $e^{*} \in E_{0}$, являющееся $C$-покрываемым относительно $E_{0}$. Пусть, кроме того, $O\left(\xi\left(\left|\mathcal{N}_{e^{*}}\left(E_{0}\right)\right|\right)\right)$ - временная сложность вспомогательной процедуры (с линейными пространственными затратами) для поиска системы представителей семейства $\mathcal{N}_{e^{*}}\left(E_{0}\right)$, имеющей длину, не превосходящую С. Тогда алгоритм ПоКРЫТИЕ РЕБЕР СПЕЦИАЛЬНЫХ ГРАФОВ РАВНЫМИ КРУГАМИ является $C$-приближенным для задачи IPGD в этом классе графов и имеет для любого $G \in \mathcal{G}$ u $r>0$ временную сложность $O((n+\varphi(n)+\xi(n)) O P T(G, r))$ и пространственные затраты $O(n)$, где $n=|E|$.

Д о к а з а т е л ь с т в о. Поскольку множество $\mathcal{N}_{r}\left(E^{\prime}\right)$ к шагу 5 становится максимальным независимым в $\mathcal{N}_{r}(E)$, то $\left|E^{\prime}\right| \leq O P T$, откуда $|H| \leq C\left|E^{\prime}\right| \leq C O P T$.

Временная сложность шага 3 равна $O(n+\varphi(n)+\xi(n))$, шагов 4 и $5-O(n)$, число повторений тела цикла по шагам 3-4 не превосходит $O P T \leq n$. Следовательно, временная сложность алгоритма имеет порядок $O((n+\varphi(n)+\xi(n)) O P T)$, при этом его пространственная сложность равна $O(n)$ с учетом линейности по $\left|\mathcal{N}_{e^{*}}\left(E_{0}\right)\right|$ пространственных затрат вспомогательной процедуры поиска множества $U\left(e^{*}, E_{0}\right)$.

Для построения на основе алгоритма ПоКРЫТИЕ РЕБЕР СПЕЦИАЛЬНЫХ ГРАФОВ РАВНЫми КРУГАми $O(1)$-приближенных алгоритмов для задачи IPGD необходимо осуществлять эффективный поиск $C$-покрываемых ребер. Ниже нами будет сформулировано достаточное условие $C$-покрываемости. Для произвольного $\mathcal{N} \subseteq \mathcal{N}_{r}(E)$ обозначим через $\mathcal{E}(\mathcal{N}) \subseteq E$ множество с условием, что $\mathcal{N}_{r}(\mathcal{E}(\mathcal{N}))=\mathcal{N}$.

Утверждение 2. Пусть $G=(V, E)$ - плоский граф и $C>0$ - некоторал константа. Тогда ребро $e^{*} \in E$-покрываемо (относительно $E$ ), если можно с помощью некоторой вспомогательной прочедуры найти такое множество $U\left(e^{*}, E\right) \subset \mathbb{R}^{2}$ мощности, не превосходящей $C$, что

$$
e \bigcap\left(N_{2 r}\left(e^{*}\right) \bigcap \bigcup_{u \in U\left(e^{*}, E\right)} N_{r}(u)\right) \neq \varnothing
$$

для любого $е \in \mathcal{E}\left(\mathcal{N}_{e^{*}}(E)\right)$.

Доказательство утверждения 2 опирается на тот факт, что $e \cap N_{2 r}\left(e^{*}\right) \neq \varnothing$ для всякого $e \in \mathcal{E}\left(\mathcal{N}_{e^{*}}(E)\right)$.

Если не накладывать никаких дополнительных ограничений либо на взаимное расположение вершин и ребер плоского графа $G$, принадлежащего некоторому классу плоских графов, либо на ориентацию или длину последних, доказать выполнение данного достаточного условия для фиксированной константы $C$ весьма сложно в случае, когда этот класс графов более или менее широк.

Основная идея реализации шага 3 в основном алгоритме. Для метрических графов, и, в частности, для графов Габриеля, графов относительных окрестностей и минимальных евклидовых остовных деревьев ограничения на взаимное расположение вершин и ребер явно или неявно вытекают из их определения. Точнее, для каждого из этих трех типов плоских 
графов выполнено следующее общее свойство: для всякого подмножества $E_{0} \subseteq E$ множества ребер $E$ произвольного графа заданного типа можно за полиномиальное время найти такое ребро $e^{*} \in E_{0}$, что выполнено соотношение $M\left(e^{*}\right) \cap V_{0}=\varnothing$, где $V_{0}-$ множество концевых вершин ребер из $E_{0}$; ориентация и расположение на плоскости множества $M\left(e^{*}\right)$ однозначно определяется ребром $e^{*}$, в то время как форма множества $M\left(e^{*}\right)$ задается типом графа.

Благодаря особенностям формы и взаимного расположения множеств $M\left(e^{*}\right)$ и $N_{2 r}\left(e^{*}\right)$ для каждого из перечисленных трех типов графов удается гарантировать (см. следующие разделы), что всякий отрезок из $\mathcal{E}\left(\mathcal{N}_{e^{*}}\left(E_{0}\right)\right)$ пересекает множество $N_{2 r}\left(e^{*}\right) \backslash M\left(e^{*}\right)$. С учетом утверждения 2 отсюда следует, что для получения системы представителей семейства $\mathcal{N}_{e^{*}}\left(E_{0}\right)$ достаточно найти такое множество $U\left(e^{*}, E_{0}\right)$ небольшой константной мощности, что

$$
N_{2 r}\left(e^{*}\right) \backslash M\left(e^{*}\right) \subset \bigcup_{u \in U\left(e^{*}, E_{0}\right)} N_{r}(u) .
$$

Следовательно, систему представителей семейства $\mathcal{N}_{e^{*}}\left(E_{0}\right)$ можно эффективно находить, строя "хорошее" частичное покрытие множества $N_{2 r}\left(e^{*}\right)$ небольшим числом кругов радиуса $r$, которое не зависит от расположения отрезков из $\mathcal{E}\left(\mathcal{N}_{e^{*}}\left(E_{0}\right)\right)$ относительно $e^{*}$. В последующих разделах описываются процедуры построения таких частичных покрытий небольшой мощности для каждого из трех перечисленных типов метрических графов.

Окончательно, предлагаемые в работе приближенные алгоритмы состоят в применении алГорИТма ПОКРЫТИЕ РЕБЕР СПЕЦИАЛЬНЫХ ГРАФОВ РАВНЫМИ КРУГАМИ, КОТОрЫЙ ВЫЗЫВаеТ на своем шаге 3 специфичную для типа графа процедуру поиска соответствующего частичного покрытия множества $N_{2 r}\left(e^{*}\right)$ мощности, не превосходящей определяемой этим типом константы $C$. Типом графа определяется также и способ, которым следует осуществлять поиск подходящего $C$-покрываемого ребра $e^{*}$.

С одной стороны, благодаря своей идейной простоте, применение вышеописанного подхода к задаче IPGD, рассматриваемой на любом из перечисленных типов метрических графов, дает существенный выигрыш в гарантированной производительности и точности по сравнению с непосредственным применением подхода на основе $\varepsilon$-сетей [1], доказавшего свою эффективность [10] при проектировании $O(1)$-приближенных алгоритмов для общей постановки задачи IPGD. C другой стороны, данный подход несет в себе и определенные недостатки, поскольку мощность построенного покрытия множества $N_{2 r}\left(e^{*}\right) \backslash M\left(e^{*}\right)$ не зависит от числа отрезков в $\mathcal{E}\left(\mathcal{N}_{e^{*}}\left(E_{0}\right)\right)$ и их расположения относительно $e^{*}$. Как следствие, мощность такого покрытия может быть велика по сравнению, например, с мощностью системы представителей, в которую включено по одной точке из каждого объекта семейства $\mathcal{N}_{e^{*}}\left(E_{0}\right)$.

Краткий обзор близких идей. В литературе известно несколько примеров построения $O(1)$-приближенных алгоритмов на основе похожих идей для близких задач, в которых, в отличие от IPGD, отрезкам допускается попарно пересекаться по относительной внутренности. При этом в такого рода задачах накладываются дополнительные ограничения либо на длину отрезков из $E$, либо на их ориентацию. Так, например, при условии, что отрезки из $E$ имеют одинаковую ориентацию, скажем, параллельную координатной оси $O x$, для задачи удается построить следующий 4-приближенный алгоритм [6], совпадающий по структуре с алГорИТмом ПОКРЫТИЕ РЕБЕР СПЕЦИАЛЬНЫХ ГРАФОВ РАВНЫМИ КРУГАМИ: ОТреЗКИ ИЗ $E$ упорядочиваются по $x$-координате их правого конца, в качестве $e^{*} \in E_{0}$ выбирается отрезок с наименьшей $x$-координатой его правого конца, при этом все отрезки из $\mathcal{E}\left(\mathcal{N}_{e^{*}}\left(E_{0}\right)\right)$ обязаны пересекать соответствующий полукруг радиуса $2 r$ с центром в этом конце; данный полукруг можно покрыть не более чем 4 кругами радиуса $r$. В [4] используется та же самая идея для случая, когда отрезки из $E$ имеют нулевую длину.

Как следует из результатов работы [7], алгоритм ПокРЫтиЕ РЕБеР СПЕцИАЛЬНЫХ ГРАФОВ РАВНЫМИ КРУГАМИ является $C$-приближенным в классе $\mathcal{G}_{\lambda}$, состоящем из всех таких плоских графов $G=(V, E)$, у которых всякое ребро $e \in E$ имеет евклидову длину, ограниченную сверху величиной $\lambda r$, где $\lambda>0$ - некоторая зависящая только от класса $\mathcal{G}_{\lambda}$ константа, 
$C$ зависит линейно от $\lambda$. В этом легко убедиться, заметив, что для всякого $e \in E$ множество $N_{2 r}(e)$ может быть полностью покрыто $O(\lambda)$ кругами радиуса $r$.

\section{12-приближенный алгоритм для графов относительных окрестностей}

В данном разделе для задачи IPGD в классе графов относительных окрестностей строится $O(1)$-приближенный алгоритм, опирающийся на алгоритм ПОКРЫТИЕ РЕБЕР СПЕЦИАЛЬНЫХ ГРАФОВ РАВНЫМИ КРУГАМИ. При этом при реализации его шага 3 используется идея, описанная в предыдущем разделе, которая заключается в построении покрытия множества $N_{2 r}(e) \backslash M(e)$ малым числом кругов радиуса $r$ для произвольно выбранного ребра $e \in E_{0}$ и некоторого специального множества $M(e)$. Следующее характеристическое свойство графов относительных окрестностей определяет специфичный для графов этого типа вид множества $M(e)$.

С в о й с т в о 1 . Пусть $G=(V, E)$ - граф относительных окрестностей, $e=\left[u_{1}, u_{2}\right] \in E$ и $M_{R N}(e)=\operatorname{int}\left(N_{2 \Delta}\left(u_{1}\right) \cap N_{2 \Delta}\left(u_{2}\right)\right)$, где $\Delta=d\left(u_{1}, u_{2}\right) / 2>0$ и $\operatorname{int} N$ обозначает внутренность подмножества $N \subset \mathbb{R}^{2}$. Тогда $M_{R N}(e) \cap V=\varnothing$.

Следующая лемма дает несколько более жесткое по сравнению с утверждением 2 достаточное условие $C$-покрываемости ребра, специфичное для графов относительных окрестностей.

Лемма 1. Пусть $G=(V, E)-$ граф относительных окрестностей и $e=\left[u_{1}, u_{2}\right] \in E$. Тогда ребро е $C$-покрываемо (относительно $E$ ), если можно за полиномиальное время найти такое не более чем $C$-элементное множество $U(e) \subset \mathbb{R}^{2}$, что

$$
N_{2 r}(e) \backslash M_{R N}(e) \subset \bigcup_{u \in U(e)} N_{r}(u) .
$$

Д о к а з а т е л ь с т в о. Очевидно, $g \cap N_{2 r}(e) \neq \varnothing$ для всякого $g \in \mathcal{E}\left(\mathcal{N}_{e}\right)$. Кроме того, $M_{R N}(e) \cap V=\varnothing$ по свойству 1 . Поскольку отрезки из $E$ пересекаются не более чем в своих концевых точках, то всякий отрезок $g \in \mathcal{E}\left(\mathcal{N}_{e}\right)$ обязан пересекать множество $N_{2 r}(e) \backslash M_{R N}(e)$. Таким образом, $g \cap N_{2 r}(e) \cap \bigcup_{u \in U(e)} N_{r}(u) \neq \varnothing$ в случае, когда включение (1) выполнено. Следовательно, по утверждению 2 ребро $e$-покрываемо относительно $E$.

Таким образом, для того, чтобы полностью определить работу алгоритма ПокРытиЕ РЕБЕР СПЕЦИАЛЬНЫХ ГРАФОВ РАВНЫМИ КРУГАМИ В КЛасСе Графов оТносИТеЛЬНЫХ окрестностей, достаточно описать для произвольного ребра $e \in E$ любого графа $G=(V, E)$ этого типа процедуру построения множества $U(e)$ мощности, не большей $C$, с условием, что для ребра $е$ и множества $U(e)$ выполнено соответствующее включение (1). Ниже дается процедура построения такого множества $U(e)$ для $C=12$.

П р о ц е д у р а ПОИСК ЧАСТИЧНОГО ПОКРЫТИЯ $2 r$-ОКРЕСТНОСТИ РЕБРА ГРАФА ОТНОСИТЕЛЬНЫХ ОКРЕСТНОСТЕЙ

Вход: $r>0$ и ребро $e=\left[u_{1}, u_{2}\right]$ графа относительных окрестностей.

Выход: множество $U_{R N}(e)$ с условием, что для $e$ и $U_{R N}(e)$ выполнено включение (1).

1. Для каждого $s \in\{1,2\}$ построить правильный шестиугольник, вписанный в круг $N_{2 r}\left(u_{s}\right)$, имеющий такую ориентацию, что прямая, проходящая через $e$, содержит пару вершин этого шестиугольника; сформировать семиточечное множество $V_{s}$, содержащее кроме точки $u_{s}$ середины сторон (длины $2 r$ ) данного шестиугольника.

2. Для каждого $s \in\{1,2\}$ выбрать подмножество $U_{s} \subset V_{s}$ с условием, что $\left|U_{s}\right|=5$ и $T_{s} \subset$ $\bigcup_{u \in U_{s}} N_{r}(u)$, где замкнутые полукруги $T_{1}$ и $T_{2}$ радиуса $2 r$ с центрами в $u_{1}$ и $u_{2}$ соответственно вместе с некоторым прямоугольником $R$ составляют в объединении объект $N_{2 r}(e)$, иными словами, $N_{2 r}(e)=T_{1} \cup T_{2} \cup R$. 
3. Если либо $\Delta \geq \frac{(2 \sqrt{3}-1) r}{2 \sqrt{4 \sqrt{3}-6}}$, либо $\Delta \in(0, r / 2]$, вернуть $U_{R N}(e):=U_{1} \cup U_{2}$.

4. При $\Delta \in\left(r / 2, \frac{(2 \sqrt{3}-1) r}{2 \sqrt{4 \sqrt{3}-6}}\right)$ выбрать пару симметричных относительно $u_{s}$ точек $v_{s 1}$, $v_{s 2} \in U_{s}, s=1,2$, и положить $a_{i}=\frac{v_{1 i}+v_{2 i}}{2}$, где $v_{1 i}$ и $v_{2 i}$ лежат по одну сторону от прямой, проходящей через $e, i=1,2$.

5. Вернуть $U_{R N}(e):=U_{1} \cup U_{2} \cup\left\{a_{1}, a_{2}\right\}$.

Следующее утверждение сообщает эффективность предложенной процедуры.

Лемма 2. Для ребра е и множества $U_{R N}($ e) соответственно на входе и на выходе процедуры ПОИСК ЧАСТИЧНОГО ПОКРЫТИЯ $2 r$-ОКРЕСТНОСТИ РЕБРА ГРАФА ОТНОСИТЕЛЬНЫХ ОКРЕСТНОСТЕЙ выполнено включение (1), причем $\left|U_{R N}(e)\right| \leq 12$.

Д о к а з а т е л ь с т в о. Очевидно, $\left|U_{R N}(e)\right| \leq 12$. Покажем выполнение включения (1), сохраняя обозначения описанной процедуры. Пусть $f_{1}(e)$ и $f_{2}(e)$ - отрезки, параллельные и равные по длине $e$, с условием, что

$$
\operatorname{bd} N_{2 r}(e)=S_{1} \cup S_{2} \cup f_{1}(e) \cup f_{2}(e),
$$

причем $f_{i}(e)$ лежит по одну сторону с отрезком $\left[v_{1 i}, v_{2 i}\right]$ и $S_{s}$ - полуокружность радиуса $2 r$ на границе $T_{s}, s=1,2$. Здесь bd $N$ обозначает границу подмножества $N \subset \mathbb{R}^{2}$.

Включение (1) выполнено, если $f_{i}(e) \cap M_{R N}(e) \cap N_{r}\left(v_{s i}\right) \neq \varnothing$ для любого $s=1,2$. Действительно, последнее равносильно неравенству $\frac{2 r^{2}}{\Delta+\sqrt{\Delta^{2}-r^{2}}} \leq r \sqrt{4 \sqrt{3}-6}$ или неравенству $\Delta \geq \frac{(2 \sqrt{3}-1) r}{2 \sqrt{4 \sqrt{3}-6}}$. Поскольку $c_{s i}=R \cap \operatorname{bd} N_{r}\left(v_{s i}\right) \cap \operatorname{bd} N_{2 r}\left(u_{s}\right) \in M_{R N}(e)$, то включение (1) справедливо. Аналогично в случае $\Delta \in(0, r / 2]$ легко убедиться в том, что справедливо более жесткое, чем (1), включение

$$
N_{2 r}(e) \subset \bigcup_{u \in U_{R N}(e)} N_{r}(u)
$$

Рассмотрим теперь случай, когда $\Delta \in\left(\frac{r}{2}, \frac{(2 \sqrt{3}-1) r}{2 \sqrt{4 \sqrt{3}-6}}\right)$. Пусть $u_{s i}=f_{i}(e) \cap \operatorname{bd} N_{r}\left(v_{s i}\right)$. В подслучае $\Delta \in\left[r, \frac{(2 \sqrt{3}-1) r}{2 \sqrt{4 \sqrt{3}-6}}\right)$ включение (1) будет установлено, если доказать, что $u_{s i}, c_{s i} \in$ $N_{r}\left(a_{i}\right)$. Действительно, $u_{s i}, c_{s i} \in N_{r}\left(v_{s i}\right)$, и, более того, $\left[c_{1 i}, c_{2 i}\right] \subset M_{R N}(e)$ в этом подслучае. Следовательно,

$$
N_{2 r}(e) \backslash\left(M_{R N}(e) \bigcup \bigcup_{u \in U_{1} \cup U_{2}} N_{r}(u)\right) \subset \bigcup_{i \in\{1,2\}} \operatorname{conv}\left\{u_{1 i}, u_{2 i}, c_{1 i}, c_{2 i}\right\} .
$$

Таким образом, для доказательства включения (1) достаточно показать, что

$$
\operatorname{conv}\left\{u_{1 i}, u_{2 i}, c_{1 i}, c_{2 i}\right\} \subset N_{r}\left(a_{i}\right) .
$$

Имеем $d\left(u_{s i}, a_{i}\right)^{2}=(\Delta-r \sqrt{4 \sqrt{3}-6})^{2}+(2-\sqrt{3})^{2} r^{2}<r^{2}$. Более того, $d\left(c_{s i}, a_{i}\right)=(\Delta-r)<r$.

В подслучае $\Delta \in(r / 2, r)$ положим $c_{s i}^{\prime}=R \cap \operatorname{bd} N_{r}\left(v_{s i}\right) \cap \mathrm{bd} N_{r}\left(u_{s}\right)$. В этом подслучае снова $\left[c_{1 i}^{\prime}, c_{2 i}^{\prime}\right] \subset M_{R N}(e)$ и

$$
N_{2 r}(e) \backslash\left(M_{R N}(e) \bigcup \bigcup_{u \in U_{1} \cup U_{2}} N_{r}(u)\right) \subset \bigcup_{i \in\{1,2\}} \operatorname{conv}\left\{u_{1 i}, u_{2 i}, c_{1 i}^{\prime}, c_{2 i}^{\prime}\right\} .
$$

Имеем $d\left(u_{s i}, a_{i}\right)^{2}<r^{2}$ и $d\left(c_{s i}^{\prime}, a_{i}\right)^{2}=\left(\Delta-\frac{r}{2}\right)^{2}+\frac{3 r^{2}}{4} \leq r^{2}$.

С учетом лемм 1 и 2 , а также утверждения 1 можно сформулировать следующий приближенный алгоритм для задачи IPGD в классе графов относительных окрестностей. 
Теорема 1. Алгоритм ПОКРЫТИЕ РЕБЕР СПЕЦИАЛЬНЫХ ГРАФОВ РАВНЫМИ КРУГАМИ, который на своем шаге 3 выбирает произвольное $e \in E_{0}$ и выполняет для этого ребра процедуру ПОИСК ЧАСТИЧНОГО ПОКРЫТИЯ $2 r$-ОКРЕСТНОСТИ РЕБРА ГРАФА ОТНОСИТЕЛЬНЫХ ОКРЕСТНОСТЕЙ, является 12-приближенным для задачи IPGD в классе графов относительных окрестностей с временной сложностью $O(n O P T)$ и затратами памяти $O(n)$, где $n=|E|$.

\section{10-приближенный алгоритм для минимальных евклидовых остовных деревьев}

Поскольку всякое минимальное евклидово остовное дерево на произвольном множестве точек $V$ в общем положении является подграфом некоторого графа относительных окрестностей на этом множестве, то для задачи IPGD в классе таких деревьев применим описанный в предыдущем разделе 12-приближенный алгоритм. В данном разделе будет показано, что для задачи IPGD в классе минимальных евклидовых остовных деревьев имеется 10-приближенный алгоритм. Этот алгоритм основан на применении той же самой идеи построения на шаге 3 алГорИТма ПОКРЫТИЕ РЕБЕР СПЕЦИАЛЬНЫХ ГРАФОВ РАВНЫМИ КРУГАМИ ПОКрЫТИЯ КрУГамИ радиуса $r$ соответствующего дополнения евклидовой $2 r$-окрестности некоторого специального ребра $е$ до множества $M_{M S T}(e)$, чей вид будет описан ниже. При этом меньшее значение константы $C$ достигается за счет того, что $M_{R N}(e) \subset M_{M S T}(e)$.

Для того чтобы описать вид множества $M_{M S T}(e)$, воспользуемся следующим простым фактом, вытекающим из определения минимального евклидова остовного дерева.

С в о й с т в о 2 . Пусть $G=(V, E)$ - минимальное евклидово остовное дерево с корнем $v_{0} \in V, \operatorname{depth}(u)=\operatorname{depth}\left(u \mid v_{0}, G\right)-$ расстояние в $G$ от $v_{0}$ до произвольной вершины $u \in V$, a $V\left(u \mid v_{0}\right)$ - подмножество таких вершин в $V$, что кратчайший (по числу ребер) путь в $G$, соединяющий их с $v_{0}$, проходит через $u$. Если ребро $e=\left[u_{1}, u_{2}\right] \in E$ таково, что $\operatorname{depth}\left(u_{1}\right)=$ $\operatorname{depth}\left(u_{2}\right)-1$, то

$$
\operatorname{int} N_{2 \Delta}\left(u_{2}\right) \cap\left(V \backslash V\left(u_{2} \mid v_{0}\right)\right)=\varnothing
$$

где $\Delta=d\left(u_{1}, u_{2}\right) / 2$.

Д ок а з а т е л ь с т в о. Пусть, от противного, найдется $v \in V \backslash V\left(u_{2} \mid v_{0}\right)$ с условием, что $d\left(v, u_{2}\right)<d\left(u_{1}, u_{2}\right)$. Учитывая, что $\operatorname{depth}\left(u_{1}\right)<\operatorname{depth}\left(u_{2}\right)$, можно сконструировать дерево меньшего, чем $G$, веса, удалив из $E$ ребро $\left[u_{1}, u_{2}\right]$ и включив в него ребро $\left[v, u_{2}\right]$.

Сформулируем достаточное условие 10-покрываемости ребра минимального евклидова остовного дерева, являющееся аналогом леммы 1. Для этого сохраним обозначения свойства 2 и прОцеДУРЫ ПОИСК ЧАСТИЧНОГО ПОКРЫТИЯ $2 r$-ОКРЕСТНОСТИ РЕБРА ГРАФА ОТНОСИТЕЛЬНЫХ ОКРЕСТНОСТЕЙ.

Лемма 3. Пусть $G_{0}=\left(V_{0}, E_{0}\right)$ - подграф без изолированных вершин некоторого минимального евклидова остовного дерева и на вершинах этого дерева задана функиия $\operatorname{depth}\left(\cdot \mid v_{0}\right)$ относительно произвольно выбранной вершины $v_{0}$. Тогда ребро $e^{*}=\left[u_{1}, u_{2}\right] \in E_{0}$ 10-покрываемо относительно $E_{0}$, если $u_{2} \in \operatorname{Arg} \max _{u \in V_{0}} \operatorname{depth}(u)$ и за полиномиальное время найдется такое, не более чем 10-точечное, множество $U\left(e^{*}\right) \subset \mathbb{R}^{2}$, что

$$
N_{2 r}\left(e^{*}\right) \backslash M_{M S T}\left(e^{*}\right) \subset \bigcup_{u \in U\left(e^{*}\right)} N_{r}(u),
$$

где $M_{M S T}\left(e^{*}\right)=\operatorname{int} N_{2 \Delta}\left(u_{2}\right) \backslash S_{2}$ и $S_{2}-$ замкнутая полуокружность радиуса $2 r$ на границе полукруга $T_{2}$.

Д о к а з а т е л ь с т в о. Воспользуемся свойством 2. В случае, когда $\Delta \leq r$, можно провести рассуждения, аналогичные содержащимся в доказательстве леммы 1 для множества $E_{0}$ вместо множества $E$. Рассмотрим случай, когда $\Delta>r$. Во-первых, по условию 
$u_{2} \in \operatorname{Arg} \max _{u \in V_{0}} \operatorname{depth}(u)$, откуда следует, что ни одно из ребер в $E_{0}$, отличных от $e^{*}$, не инцидентно $u_{2}$. Во-вторых, отрезки из $E_{0}$ пересекаются не более чем в своих концевых точках. Следовательно, $e \cap\left(N_{2 r}\left(e^{*}\right) \backslash \operatorname{int} N_{2 \Delta}\left(u_{2}\right)\right) \neq \varnothing$ для любого ребра $e \in \mathcal{E}\left(\mathcal{N}_{e^{*}}\left(E_{0}\right)\right)$ с условием, что $e \cap S_{2}=\varnothing$.

Для того чтобы полностью описать работу алгоритма ПоКРЫтИЕ РЕБЕР СПЕЦИАЛЬНЫХ ГРАФОВ РАВНЫМИ КРУГАМИ в классе минимальных евклидовых остовных деревьев, основываясь на лемме 3 , опишем в ее обозначениях следующую эвристику выбора ребра $e^{*}$ на шаге 3 этого алгоритма. $\mathrm{K}$ этому шагу имеем некоторый подграф $G_{0}=\left(V_{0}, E_{0}\right)$ без изолированных вершин минимального евклидова остовного дерева $G=(V, E)$. При этом предварительно на шаге 1 алгоритма ПоКРЫТИЕ РЕБЕР СПЕцИАЛЬНЫХ ГРАФОВ РАВНЫМИ КРУГАМИ предлагается обойти исходное минимальное евклидово остовное дерево $G$ поиском в ширину за время $O(|E|)$ и вычислить расстояние $\operatorname{depth}(u)=\operatorname{depth}\left(u \mid v_{0}, G\right)$ от всякой вершины $u$ в $G$ до произвольно выбранного фиксированного корня $v_{0} \in V$ по ребрам в $G$.

П р о ц е д у р а ВЫБОР 10-ПОКРЫВАЕМОГО РЕБРА

При выполнении шага 3 за время $O\left(\left|E_{0}\right|\right)$ следует выбрать ребро $e^{*}=\left[u_{1}, u_{2}\right] \in E_{0}$ с условием, что $u_{2} \in \operatorname{Arg} \max _{u \in V_{0}} \operatorname{depth}(u)$.

Сохраняя обозначения процедуры ПОИСК чАСТИчНОГО ПОКРЫТИя $2 r$-ОКРЕСТНОСТИ РЕБРА ГРАФА ОТНОСИТЕЛЬНЫХ ОКРЕСТНОСТЕЙ и доказатеЛЬства ЛеммЫ 2, сформулируем теперь вспомогательную процедуру поиска не более чем 10-элементного множества $U_{M S T}\left(e^{*}\right)$, для которого выполнено включение (2) относительно отрезка $e^{*}$, найденного согласно эвристике ВЫБОР 10-ПОКРЫВАЕМОГО РЕБРА.

П р о ц е д у р а ПОИСК ЧАСТИЧНОГО ПОКРЫТИЯ $2 r$-ОКРЕСТНОСТИ РЕБРА МИНИМАЛЬНОГО ЕВКЛИДОВА ОСТОВНОГО ДЕРЕВА

Вход: $r>0$ и ребро $e^{*}=\left[u_{1}, u_{2}\right]$ произвольного подграфа $G_{0}=\left(V_{0}, E_{0}\right)$ без изолированных вершин некоторого минимального евклидова остовного дерева, причем $u_{2} \in \operatorname{Arg} \max _{u \in V_{0}} \operatorname{depth}(u)$. Выход: множество $U_{M S T}\left(e^{*}\right)$, для которого выполнено включение $(2)$ относительно $e^{*}$.

1. Построить множество $U_{1}$ так же, как и в процедуре Поиск чАстичного ПокРытия $2 r$-ОКРЕСТНОСТИ РЕБРА ГРАФА ОТНОСИТЕЛЬНЫХ ОКРЕСТНОСТЕЙ.

2. При $\Delta \in(0, r / 2]$ вернуть $U_{M S T}\left(e^{*}\right):=U_{1} \cup U_{2}$, где $U_{2}$ построено так же, как и в процеДУре ПОИСК ЧАСТИЧНОГО ПОКРЫТИЯ $2 r$-ОКРЕСТНОСТИ РЕБРА ГРАФА ОТНОСИТЕЛЬНЫХ ОКРЕСТНОСТЕЙ.

3. Составить множество $U_{2}^{\prime}$ из середин трех целиком содержащихся в $T_{2}$ сторон соответствующего правильного шестиугольника, вписанного в круг $N_{2 r}\left(u_{2}\right)$.

4. Если $\Delta \geq \frac{(2 \sqrt{3}-1) r}{2 \sqrt{4 \sqrt{3}-6}}$, то вернуть $U_{M S T}\left(e^{*}\right):=U_{1} \cup U_{2}^{\prime}$.

5. При $\Delta \in\left[r, \frac{(2 \sqrt{3}-1) r}{2 \sqrt{4 \sqrt{3}-6}}\right)$ положить $z_{i}:=f_{i}\left(e^{*}\right) \cap \operatorname{bd} N_{2 \Delta}\left(u_{2}\right)$ и $a_{i}^{\prime}:=\frac{u_{1 i}+z_{i}}{2}, i=1,2$ (см. обозначения доказательства леммы 2$)$.

6. Для $\Delta \in(r / 2, r)$ в прямоугольной системе координат с началом в $u_{2}$, у которой ось $O x$ направлена вдоль $e^{*}$, а ось $O y$ направлена в сторону отрезка $f_{i}\left(e^{*}\right)$, положить $a_{i}^{\prime}=\frac{u_{1 i}+b_{i}}{2}$, где $b_{i}=(0,2 \Delta)$.

7. Вернуть $U_{M S T}\left(e^{*}\right):=U_{1} \cup U_{2}^{\prime} \cup\left\{a_{1}^{\prime}, a_{2}^{\prime}\right\}$.

Следующая лемма сообщает эффективность процедуры Поиск чАСТичного ПокРЫтия $2 r$-ОКРЕСТНОСТИ РЕБРА МИНИМАЛЬНОГО ЕВКЛИДОВА ОСТОВНОГО ДЕРЕВА.

Лемма 4. Процедура ПОИСК ЧАСТИЧНОГО ПОКРЫТИЯ $2 r$-ОКРЕСТНОСТИ РЕБРА МИНИМАЛЬНОГО ЕВКЛИДОВА ОСТОВНОГО ДЕРЕВА находит такое, не более чем 10-точечное, множество $U_{M S T}\left(e^{*}\right)$, что для него выполнено включение (2), где $e^{*}-$ ребро на входе этой проиедурь. 
Д о к а з а т е л ь с т в о. При $\Delta \geq \frac{(2 \sqrt{3}-1) r}{2 \sqrt{4 \sqrt{3}-6}}$ можно показать аналогично тому, как это делалось в доказательстве леммы 2 , что $N_{2 r}\left(e^{*}\right) \backslash M_{M S T}\left(e^{*}\right) \subset \underset{u \in U_{M S T}\left(e^{*}\right)}{\bigcup} N_{r}(u)$. Если $\Delta \in$ $(0, r / 2]$, то по аналогии с доказательством леммы 2 имеем, что $N_{2 r}\left(e^{*}\right) \subset \underset{u \in U_{M S T}\left(e^{*}\right)}{\bigcup} N_{r}(u)$.

При $\Delta \in\left[r, \frac{(2 \sqrt{3}-1) r}{2 \sqrt{4 \sqrt{3}-6}}\right)$ достаточно показать, что точки $u_{1 i}, z_{i}$ и $c_{1 i}=R \cap \operatorname{bd} N_{2 r}\left(u_{1}\right) \cap$ $\operatorname{bd} N_{r}\left(v_{1 i}\right)$ лежат в круге $N_{r}\left(a_{i}^{\prime}\right)$, поскольку в этом случае

$$
N_{2 r}\left(e^{*}\right) \backslash\left(M_{M S T}\left(e^{*}\right) \bigcup \bigcup_{u \in U_{1} \cup U_{2}^{\prime}} N_{r}(u)\right) \subset \bigcup_{i=1,2} \operatorname{conv}\left\{u_{1 i}, z_{i}, c_{1 i}\right\} .
$$

Имеем, подставляя $\Delta=r$ и $\Delta=\frac{(2 \sqrt{3}-1) r}{2 \sqrt{4 \sqrt{3}-6}}$ в выражение ниже,

$$
d\left(u_{1 i}, a_{i}^{\prime}\right)^{2}=d\left(z_{i}, a_{i}^{\prime}\right)^{2}=\left(\frac{r^{2}}{\Delta+\sqrt{\Delta^{2}-r^{2}}}-\frac{r \sqrt{4 \sqrt{3}-6}}{2}\right)^{2}<r^{2} .
$$

Кроме того,

$$
d\left(c_{1 i}, a_{i}^{\prime}\right)^{2}=\left(r-\frac{\sqrt{4 \sqrt{3}-6} r}{2}-\frac{r^{2}}{\Delta+\sqrt{\Delta^{2}-r^{2}}}\right)^{2}+(2-\sqrt{3})^{2} r^{2}<r^{2} .
$$

При $\Delta \in(r / 2, r)$ введем обозначения. Пусть $z_{i}^{\prime}=(0,2 r)$ и $c_{i}^{\prime}-$ точка в пересечении $\mathrm{bd} N_{r}\left(v_{1 i}\right) \cap \operatorname{bd} N_{2 \Delta}\left(u_{2}\right)$ с наибольшей $y$-координатой. Для обоснования включения (2) достаточно убедиться в том, что

$$
\operatorname{conv}\left\{u_{1 i}, z_{i}^{\prime}, b_{i}, c_{i}^{\prime}\right\} \subset N_{r}\left(a_{i}^{\prime}\right) .
$$

Очевидно, $a_{i}^{\prime}=\left(\Delta-\frac{r \sqrt{4 \sqrt{3}-6}}{2}, r+\Delta\right)$. Также имеем с учетом того, что $\Delta \in(r / 2, r)$ :

$$
d\left(u_{1 i}, a_{i}^{\prime}\right)^{2}=d\left(z_{i}^{\prime}, a_{i}^{\prime}\right)^{2}=d\left(b_{i}, a_{i}^{\prime}\right)^{2}=\left(\Delta-\frac{r \sqrt{4 \sqrt{3}-6}}{2}\right)^{2}+(r-\Delta)^{2}<r^{2} .
$$

Используя теорему косинусов, выпишем координаты $c_{i}^{\prime}$ :

$$
x_{c_{i}^{\prime}}=2 \Delta-\frac{4 r^{2} \Delta+\sqrt{3} r^{2} \sqrt{4 \Delta^{2}-r^{2}}}{4 \Delta^{2}+3 r^{2}}, \quad y_{c_{i}^{\prime}}=\frac{2 \Delta r \sqrt{4 \Delta^{2}-r^{2}}+4 \sqrt{3} r \Delta^{2}+\sqrt{3} r^{3}}{4 \Delta^{2}+3 r^{2}} .
$$

Оценим отдельно $\left|x_{c_{i}^{\prime}}-x_{a_{i}^{\prime}}\right|$ и $\left|y_{c_{i}^{\prime}}-y_{a_{i}^{\prime}}\right|$.

Начнем с $\left|x_{c_{i}^{\prime}}-x_{a_{i}^{\prime}}\right|$. Покажем, что $x_{c_{i}^{\prime}} \geq x_{a_{i}^{\prime}}$. Имеем, что

$$
Q_{x}=x_{c_{i}^{\prime}}-x_{a_{i}^{\prime}} \geq \frac{4 \Delta^{3}-r^{2} \Delta+8 r \Delta^{2} / 5+6 r^{3} / 5-\sqrt{3} r^{2} \sqrt{4 \Delta^{2}-r^{2}}}{4 \Delta^{2}+3 r^{2}} .
$$

Легко убедиться в том, что $Q_{1}=-r^{2} \Delta+8 r \Delta^{2} / 5 \geq-5 r^{3} / 32$ при $\Delta \in(r / 2, r)$. С другой стороны, применяя дифференциальное исчисление, получаем $Q_{2}=4 \Delta^{3}-\sqrt{3} r^{2} \sqrt{4 \Delta^{2}-r^{2}} \geq-0.35 r^{3}$ в той же области изменения $\Delta$. Подставляя эти оценки для $Q_{1}$ и $Q_{2}$ в выражение $(3)$, получаем, что $x_{c_{i}^{\prime}} \geq x_{a_{i}^{\prime}}$.

Производную $\frac{d Q_{x}}{d \Delta}$ после приведения подобных слагаемых можно записать в виде:

$$
\frac{d Q_{x}}{d \Delta}=1-\frac{4 r^{2}}{4 \Delta^{2}+3 r^{2}}-\frac{4 r^{2}\left(\frac{\sqrt{3}\left(5 r^{2}-4 \Delta^{2}\right) \Delta}{\sqrt{4 \Delta^{2}-r^{2}}}-8 \Delta^{2}\right)}{\left(4 \Delta^{2}+3 r^{2}\right)^{2}} .
$$


Покажем, что $\frac{d Q_{x}}{d \Delta}$ возрастает по $\Delta$ и имеет единственный ноль, являющийся минимумом $Q_{x}$. Для этого достаточно показать, что $\frac{d Q_{x}}{d \Delta}$ отрицательна при $\Delta \rightarrow r / 2$, положительна для $\Delta=r$, а также что производная по $\Delta$ от выражения $Z=\frac{\frac{\sqrt{3}\left(5 r^{2}-4 \Delta^{2}\right) \Delta}{\sqrt{4 \Delta^{2}-r^{2}}}-8 \Delta^{2}}{\left(4 \Delta^{2}+3 r^{2}\right)^{2}}$ не положительна при $\Delta \in(r / 2, r)$. Последняя производная равна

$$
\frac{64 \Delta^{3}-48 \Delta r^{2}+\frac{\sqrt{3}}{\left(4 \Delta^{2}-r^{2}\right)^{3 / 2}}\left(128 \Delta^{6}+96 \Delta^{2} r^{4}-432 \Delta^{4} r^{2}-15 r^{6}\right)}{\left(4 \Delta^{2}+3 r^{2}\right)^{3}} .
$$

Дифференцируя по $\Delta$, получаем, что $P_{1}=128 \Delta^{6}+96 \Delta^{2} r^{4}-432 \Delta^{4} r^{2}-15 r^{6} \leq-16 r^{6}$ для $\Delta \in(r / 2,11 r / 16]$ и $P_{1} \leq-52 r^{6}$ для $\Delta \in(11 r / 16, r)$. Кроме того, $64 \Delta^{3}-48 \Delta r^{2} \leq 16 r^{3}$ при $\Delta \in(r / 2, r)$, откуда следует, что $\frac{d Z}{d \Delta} \leq 0$.

Таким образом, окончательно получаем, что максимум $\left|Q_{x}\right|$ совпадает с максимальным из значений $Q_{x}$ при $\Delta=r / 2$ и $\Delta=r$, откуда $\left|Q_{x}\right| \leq r / 2$.

Теперь оценим $\left|y_{c_{i}^{\prime}}-y_{a_{i}^{\prime}}\right|$. Покажем, что $y_{a_{i}^{\prime}} \geq y_{c_{i}^{\prime}}$. Поскольку

$$
Q_{y}=y_{a_{i}^{\prime}}-y_{c_{i}^{\prime}}=\frac{4 \Delta^{3}-2 \Delta r \sqrt{4 \Delta^{2}-r^{2}}+3 r^{2} \Delta+4 \Delta^{2} r+3 r^{3}-4 \sqrt{3} r \Delta^{2}-\sqrt{3} r^{3}}{4 \Delta^{2}+3 r^{2}},
$$

в этом легко убедиться, доказав неотрицательность каждого из двух выражений $Q_{1}^{\prime}=4 \Delta^{3}-$ $2 \Delta r \sqrt{4 \Delta^{2}-r^{2}}$ и $Q_{2}^{\prime}=3 r^{2} \Delta+4 \Delta^{2} r+3 r^{3}-4 \sqrt{3} r \Delta^{2}-\sqrt{3} r^{3}$ при $\Delta \in(r / 2, r)$.

Запишем $Q_{y}$ в более удобной форме:

$$
Q_{y}=\Delta+r-\sqrt{3} r+\frac{2 \sqrt{3} r^{3}-2 r \Delta \sqrt{4 \Delta^{2}-r^{2}}}{4 \Delta^{2}+3 r^{2}}
$$

Продифференцировав по $\Delta$ эту разность, имеем

$$
\frac{d Q_{y}}{d \Delta}=1-\frac{2 r^{3}}{\left(4 \Delta^{2}+3 r^{2}\right)^{2}}\left(\frac{28 \Delta^{2}-3 r^{2}}{\sqrt{4 \Delta^{2}-r^{2}}}+8 \sqrt{3} \Delta\right)
$$

Тогда

$$
=\frac{2 r^{3}}{\left(4 \Delta^{2}+3 r^{2}\right)^{2}}\left[\frac{1}{\sqrt{4 \Delta^{2}-r^{2}}}\left(\frac{\left(28 \Delta^{2}-3 r^{2}\right) 16 \Delta}{4 \Delta^{2}+3 r^{2}}-\frac{4 \Delta\left(28 \Delta^{2}-11 r^{2}\right)}{4 \Delta^{2}-r^{2}}\right)+8 \sqrt{3}\left(\frac{16 \Delta^{2}}{4 \Delta^{2}+3 r^{2}}-1\right)\right] .
$$

Второе слагаемое в квадратных скобках неотрицательно при $\Delta \geq r / 2$. При $\Delta \geq \sqrt{7 / 12} r$ первое слагаемое в скобках неотрицательно, поскольку $\Delta^{2}+3 / 4 r^{2} \leq 4 \Delta^{2}-r^{2}$. Если же $\Delta \in[\sqrt{11 / 28} r, \sqrt{7 / 12} r)$, приводя к общему знаменателю в первом слагаемом, получаем его неотрицательность. Наконец, для $\Delta \in(r / 2, \sqrt{11 / 28} r)$ это слагаемое, очевидно, неотрицательно.

Таким образом, $\frac{d Q_{y}}{d \Delta}$ монотонно возрастает на $(r / 2, r)$, принимая отрицательные значения при $\Delta \rightarrow r / 2$. Но тогда для оценки $\left|Q_{y}\right|$ сверху достаточно подсчитать значения $Q_{y}$ при $\Delta \in$ $\{r / 2, r\}$. Имеем, что $\left|Q_{y}\right| \leq \frac{3-\sqrt{3}}{2} r$. Окончательно получаем оценку $d\left(c_{i}^{\prime}, a_{i}^{\prime}\right)^{2} \leq r^{2}$.

Из лемм 3 и 4 вытекает следующий 10-приближенный алгоритм покрытия ребер минимальных евклидовых остовных деревьев. 
Теорема 2. Алгоритм ПОКРЫТИЕ РЕБЕР СПЕЦИАЛЬНЫХ ГРАФОВ РАВНЫМИ КРУГАМИ, который на своем шаге 3 выбирает $e^{*} \in E_{0}$ согласно эвристике ВЫБОР 10-ПОКРЫВАЕМОГО РЕБРА $и$ выполняет для него процедуру ПОИСК ЧАСТИЧНОГО ПОКРЫТИЯ $2 r$-ОКРЕСТНОСТИ РЕБРА МИНИМАЛЬНОГО ЕВКЛИДОВА ОСТОВНОГО ДЕРЕВА, является 10-приближенным для задачи IPGD в классе минимальных евклидовых остовных деревъев с временной сложсностью $O(n O P T)$ и затратами памяти $O(n)$, где $n=|E|$.

\section{14-приближенный алгоритм для графов Габриеля}

В данном разделе на основе алгоритма ПоКРЫТИЕ РЕБЕР СПЕЦИАЛЬНЫХ ГРАФОВ РАВНЫми КРУГАми будет построен соответствующий 14-приближенный алгоритм для задачи IPGD в классе графов Габриеля, вызывающий вспомогательную процедуру поиска покрытия множества $N_{2 r}(e) \backslash M_{G}(e)$ для произвольного выбранного на шаге 3 ребра $e$ и определяемого им множества $M_{G}(e)$. Сформулируем удобное для дальнейших рассуждений достаточное условие 14-покрываемости ребра графа Габриеля, описывающее вид множества $M_{G}(e)$. Его доказательство аналогично доказательству леммы 1.

Лемма 5. Пусть $G=(V, E)-$ граф Габриеля, $e=\left[u_{1}, u_{2}\right] \in E u M_{G}(e)=\operatorname{int} N_{\Delta}\left(\frac{u_{1}+u_{2}}{2}\right)$, где $\Delta=\frac{d\left(u_{1}, u_{2}\right)}{2}>0$. Тогда е 14-покрываемо, если найдется такое, не более чем 14-точечное, множество $U(e) \subset \mathbb{R}^{2}$, что

$$
N_{2 r}(e) \backslash M_{G}(e) \subset \bigcup_{u \in U(e)} N_{r}(u)
$$

Сохраняя обозначения процедуры Поиск ЧАСТичНОГО ПОКРЫТИя $2 r$-ОКРЕСТНОСТИ РЕБРА ГРАФА ОТНОСИТЕЛЬНЫХ ОКРЕСТНОСТЕЙ и доказаТеЛЬства ЛеммЫ 2, сформулируем следующую процедуру.

П р о ц е д у р а ПОИСК ЧАСТИЧНОГО ПОКРЫТИЯ $2 r$-ОКРЕСТНОСТИ РЕБРА ГРАФА ГАБРИЕЛЯ

Вход: $r>0$ и произвольное ребро $e=\left[u_{1}, u_{2}\right]$ некоторого графа Габриеля.

Выход: множество $U_{G}(e)$, для которого выполнено включение (4) относительно ребра $e$.

1. Построить множества $U_{s}, s=1,2$, так же, как и в процедуре Поиск чАстичного ПОКРЫТИЯ $2 r$-ОКРЕСТНОСТИ РЕБРА ГРАФА ОТНОСИТЕЛЬНЫХ ОКРЕСТНОСТЕЙ.

2. Если $\Delta \geq \frac{(2 \sqrt{3}-1) r}{\sqrt{4 \sqrt{3}-6}}$ или $\Delta \in(0, r / 2]$, вернуть $U_{G}(e):=U_{1} \cup U_{2}$.

3. При $\Delta \in\left[2 r, \frac{(2 \sqrt{3}-1) r}{\sqrt{4 \sqrt{3}-6}}\right)$ положить $u_{0}:=\frac{u_{1}+u_{2}}{2}$ и найти две точки $z_{1 i}$ и $z_{2 i}$ в пересечении $f_{i}(e) \cap \operatorname{bd} M_{G}(e)$, где $z_{s i}$ расположена ближе к $u_{s i}$, чем $z_{(3-s) i}$; положить $a_{s i}=\frac{u_{s i}+z_{s i}}{2}$.

4. Если же $\Delta \in(r, 2 r)$, рассмотреть прямоугольную систему координат с началом в $u_{s}$ с $x$-осью, направленной вдоль $e$ в направлении к $u_{3-s}$, и $y$-осью, идущей перпендикулярно $x$-оси в направлении к отрезку $f_{i}(e)$; положить $b_{i}=(\Delta, \Delta)$ и $a_{s i}=\frac{u_{s i}+b_{i}}{2}$.

5. При $\Delta \in(r / 2, r]$ положить $a_{1 i}:=(\Delta, \sqrt{3} r)$ и $a_{2 i}:=(\Delta, \sqrt{3} r / 2)$.

6. Вернуть $U_{G}(e):=U_{1} \cup U_{2} \cup\left\{a_{s i}\right\}_{s, i=1,2}$.

Сформулируем лемму, гарантирующую эффективность процедуры Поиск чАСТичноГО ПОКРЫТИЯ $2 r$-ОКРЕСТНОСТИ РЕБРА ГРАФА ГАБРИЕЛЯ.

Лемма 6. Для ребра е и множества $U_{G}($ е) соответственно на входе и выходе процеду$p \leadsto$ ПОИСК ЧАСТИЧНОГО ПОКРЫТИЯ $2 r$-ОКРЕСТНОСТИ РЕБРА ГРАФА ГАБРИЕЛЯ выполнено включение (4), при этом $\left|U_{G}(e)\right| \leq 14$. 
Д о к а з а т е л ь с т в о случая $\Delta \geq \frac{(2 \sqrt{3}-1) r}{\sqrt{4 \sqrt{3}-6}}$ проводится так же, как и в лемме 2 с точностью до замены $2 \Delta$ на $\Delta$. Случай $\Delta \leq r / 2$ разбирается так же, как и в доказательстве этой леммы.

Случаи $\Delta \in\left[2 r, \frac{(2 \sqrt{3}-1) r}{\sqrt{4 \sqrt{3}-6}}\right)$ и $\Delta \in(r, 2 r)$ разбираются аналогично доказательству леммы 4 с точностью до замены $2 \Delta$ на $\Delta$.

Пусть, наконец, $\Delta \in(r / 2, r]$. В этом случае $d\left(u_{s i}, a_{1 i}\right)^{2}=(\Delta-r \sqrt{4 \sqrt{3}-6})^{2}+(2-\sqrt{3})^{2} r^{2}<r^{2}$ и $d\left(c_{s i}^{\prime}, a_{1 i}\right)^{2}=(\Delta-r / 2)^{2}+3 r^{2} / 4 \leq r^{2}$. Кроме того, $d\left(c_{s i}^{\prime}, a_{2 i}\right)=\Delta-r / 2<r$ и $d\left(u_{0}, a_{2 i}\right)=$ $\sqrt{3} r / 2<r$. Таким образом, $\operatorname{conv}\left\{u_{1 i}, u_{2 i}, c_{1 i}^{\prime}, c_{2 i}^{\prime}\right\} \subset N_{r}\left(a_{1 i}\right)$ и conv $\left\{c_{1 i}^{\prime}, c_{2 i}^{\prime}, u_{0}\right\} \subset N_{r}\left(a_{2 i}\right)$, откуда следует, что

$$
N_{2 r}(e) \backslash\left(M_{G}(e) \bigcup \bigcup_{u \in U_{1} \cup U_{2}} N_{r}(u)\right) \subset \bigcup_{u \in\left\{a_{s i}\right\}_{s, i=1,2}} N_{r}(u)
$$

Окончательно можно сформулировать следующий приближенный алгоритм для задачи IPGD в классе графов Габриеля.

Теорема 3. Алгоритм ПОКРЫТИЕ РЕБЕР СПЕЦИАЛЬНЫХ ГРАФОВ РАВНЫМИ КРУГАМИ, который на своем шаге 3 выбирает произвольное ребро $е \in E_{0}$ и выполняет для него прочеӘуру ПОИСК ЧАСТИЧНОГО ПОКРЫТИЯ $2 r$-ОКРЕСТНОСТИ РЕБРА ГРАФА ГАБРИЕЛЯ, является 14-приближенным для задачи IPGD в классе графов Габриеля с временной сложностью $O(n O P T)$ и затратами памяти $O(n)$, где $n=|E|$.

\section{6. Анализ достижимости верхних оценок фактора аппроксимации алгоритмов}

Приведем серию примеров задачи IPGD, на которых константы в верхних оценках факторов аппроксимации предлагаемых алгоритмов не слишком сильно отличаются от отношения $\frac{\left|H_{\mathcal{A}}(G, r)\right|}{O P T(G, r)}$, где $H_{\mathcal{A}}(G, r)$ - допустимое решение задачи IPGD для графа $G$ и радиуса $r$, выдаваемое алгоритмом, а $O P T=O P T(G, r)$ - ее оптимум.

Для произвольного плоского графа $G$ выберем $r>0$ столь малым, чтобы задача IPGD была эквивалентной задаче вершинного покрытия $G$. При этом множество $E^{\prime}$, получаемое по окончании работы алгоритма ПОКРЫТИЕ РЕБЕР СПЕЦИАЛЬНЫХ ГРАФОВ РАВНЫМИ КРУГАМИ, будет совпадать с максимальным по включению паросочетанием в $G$ и $\left|E^{\prime}\right| \leq O P T \leq 2\left|E^{\prime}\right|$. Следовательно, соотношение между длиной выдаваемого алгоритмом ПокРытиЕ РеБеР СПЕЦИАЛЬНЫХ ГРАФОВ РАВНЫМИ КРУГАМИ множества $H_{\mathcal{A}}(G, r)$ и $O P T$ будет не меньше, чем 5 , 5 и 4 для графов относительных окрестностей, графов Габриеля и минимальных евклидовых остовных деревьев соответственно.

\section{Заключение}

В работе строятся приближенные алгоритмы с константным фактором аппроксимации для задачи оптимального пересечения ребер специальных метрических графов минимальным числом одинаковых кругов. Точнее, основываясь на специфике взаимного расположения ребер и вершин графов относительных окрестностей, минимальных евклидовых остовных деревьев и графов Габриеля, построены алгоритмы с факторами аппроксимации соответственно 12, 10 и 14, сочетающие относительно невысокую трудоемкость с достаточно высокой точностью, т. е. небольшим фактором аппроксимации по сравнению с таковыми в алгоритмах для близких задач (см., например, [10]). 


\section{СПИСОК ЛИТЕРАТУРЫ}

1. Agarwal P., Pan J. Near-linear algorithms for geometric hitting sets and set covers // Proc. of 30th Ann. Symp. on Comput. Geom. 2014. P. 271-279.

2. Alon N. A Non-linear lower bound for planar epsilon-nets // Discr. Comput. Geom. 2012. Vol. 47, no. 2. P. 235-244. doi: $10.1007 / \mathrm{s} 00454-010-9323-7$.

3. Antunes D., Mathieu C., Mustafa N. Combinatorics of local search: An optimal 4-local Hall's theorem for planar graphs // Proc. of 25th Ann. Eur. Symp. on Alg. (ESA). 2017. No. 87. P. 8:1-8:13. doi: 10.4230/LIPIcs.ESA.2017.8.

4. Biniaz A., Liu P., Maheshwari A., Smid M. Approximation algorithms for the unit disk cover problem in 2D and 3D // Comput. Geom. 2017. Vol. 60. P. 8-18. doi: 10.1016/j.comgeo.2016.04.002 .

5. Bus N., Garg S., Mustafa N., Ray S. Limits of local search: Quality and efficiency // Discr. Comput. Geom. 2017. Vol. 57, no. 3. P. 607-624. doi: 10.1007/s00454-016-9819-x.

6. Dash D., Bishnu A., Gupta A., Nandy S. C. Approximation algorithms for deployment of sensors for line segment coverage in wireless sensor networks // Wireless Networks. 2013. Vol. 19, no. 5. P. 857-870. doi: 10.1007/s11276-012-0506-4 .

7. Efrat A., Katz M.J., Nielsen F., Sharir M. Dynamic data structures for fat objects and their applications // Comput. Geom. 2000. Vol. 15. P. 215-227. doi: 10.1016/S0925-7721(99)00059-0 .

8. Jaromczyk J. W., Toussaint G. T. Relative neighborhood graphs and their relatives // Proc. IEEE. 1992. Vol. 80, no. 9. P. 1502-1517. doi: 10.1109/5.163414.

9. Kobylkin K. S. Stabbing line segments with disks: Complexity and approximation algorithms // Lect. Notes Comp. Sci. 2017. Vol. 10716. P. 356-367. doi: 10.1007/978-3-319-73013-4_33.

10. Kobylkin K.S. Constant factor approximation for intersecting line segments with disks // Lect. Notes Comp. Sci. 2019. Vol. 11353. P. 447-454. doi: 10.1007/978-3-030-05348-2_39.

11. Matula D. W., Sokal R. R. Properties of Gabriel graphs relevant to geographic variation research and clustering of points in the plane // Geogr. Anal. 1980. Vol. 12, no. 3. P. 205-222. doi: $10.1111 / \mathrm{j} .1538-4632.1980 . t b 00031 . x$.

12. Madireddy R. R., Mudgal A. Stabbing line segments with disks and related problems // Proc. of 28th Canadian Conf. on Comput. Geom. 2016. P. 201-207.

13. Mustafa N., Ray S. Improved results on geometric hitting set problems // Discr. Comput. Geom. 2010. Vol. 44, no. 4. P. 883-895.

14. Pyrga E., Ray S. New existence proofs for $\varepsilon$-nets // Proc. of 24th Ann. Symp. on Comput. Geom. 2008. P. 199-207. doi: 10.1145/1377676.1377708.

Поступила 19.11.2018

После доработки 23.01.2019

Принята к публикации 4.02.2019

Кобылкин Константин Сергеевич

канд. физ.-мат. наук, старший науч. сотрудник

Институт математики и механики им. Н.Н. Красовского УрО РАН;

Уральский федеральный университет

г. Екатеринбург

e-mail: kobylkinks@gmail.com .

\section{REFERENCES}

1. Agarwal P., Pan J. Near-Linear Algorithms for Geometric Hitting Sets and Set Covers. Proc. of 30th Ann. Symp. on Comput. Geom., 2014, pp. 271-279.

2. Alon N. A Non-linear Lower Bound for Planar Epsilon-nets. Discr. Comput. Geom., 2012, vol. 47, no. 2, pp. 235-244. doi: 10.1007/s00454-010-9323-7.

3. Antunes D., Mathieu C., Mustafa N. Combinatorics of Local Search: An Optimal 4-Local Hall's Theorem for Planar Graphs. Proc. of 25th Ann. Eur. Symp. on Alg. (ESA), 2017, vol. 87, pp. 8:1-8:13. doi: 10.4230/LIPIcs.ESA.2017.8.

4. Biniaz A., Liu P., Maheshwari A., Smid M. Approximation algorithms for the unit disk cover problem in 2D and 3D. Comput. Geom., 2017, vol. 60, pp. 8-18. doi: 10.1016/j.comgeo.2016.04.002 . 
5. Bus N., Garg S., Mustafa N., Ray S. Limits of Local Search: Quality and Efficiency. Discr. Comput. Geom., 2017, vol. 57, no. 3, pp. 607-624. doi: 10.1007/s00454-016-9819-x .

6. Dash D., Bishnu A., Gupta A., Nandy S.C. Approximation algorithms for deployment of sensors for line segment coverage in wireless sensor networks. Wireless Networks, 2013, vol. 19, no. 5, pp. 857-870. doi: $10.1007 / \mathrm{s} 11276-012-0506-4$.

7. Efrat A., Katz M.J., Nielsen F., Sharir M. Dynamic data structures for fat objects and their applications. Comput. Geom., 2000, vol. 15, no. 4, pp. 215-227. doi: 10.1016/S0925-7721(99)00059-0.

8. Jaromczyk J.W., Toussaint G.T. Relative neighborhood graphs and their relatives. Proc. IEEE, 1992, vol. 80, no. 9, pp. 1502-1517. doi: 10.1109/5.163414.

9. Kobylkin K.S. Stabbing Line Segments with Disks: Complexity and Approximation Algorithms. Lect. Notes in Comp. Sci., 2017, vol. 10716, pp. 356-367. doi: 10.1007/978-3-319-73013-4_33.

10. Kobylkin K.S. Constant factor approximation for intersecting line segments with disks. Lect. Notes in Comp. Sci., 2019, vol. 11353, pp. 447-454. doi: 10.1007/978-3-030-05348-2_39.

11. Matula D.W., Sokal R.R. Properties of Gabriel graphs relevant to geographic variation research and clustering of points in the plane. Geogr. Anal., 1980, vol. 12, no. 3, pp. 205-222. doi: 10.1111/j.1538-4632.1980.tb00031.x .

12. Madireddy R.R., Mudgal A. Stabbing line segments with disks and related problems. Proc. of 28th Canadian Conf. Comput. Geom., 2016, pp. 201-207.

13. Mustafa N., Ray S. Improved Results on Geometric Hitting Set Problems. Discr. Comput. Geom., 2010, vol. 44, no. 4, pp. 883-895.

14. Pyrga E., Ray S. New Existence Proofs for E-nets. Proc. of 24th Ann. Symp. on Comput. Geom., 2008, pp. 199-207. doi: 10.1145/1377676.1377708.

Received November 19, 2018

Revised January 23, 2019

Accepted February 4, 2019

Funding Agency: This work was supported by the Russian Science Foundation (project no. 1411-00109).

Konstantin Sergeevich Kobylkin, Cand. Sci. (Phys.-Math.), Krasovsky Institute of Mathematics and Mechanics of the Ural Branch of the Russian Academy of Sciences, Ekaterinburg, 620108 Russia; Ural Federal University, Yekaterinburg, 620002 Russia, e-mail: kobylkinks@gmail.com. 